\title{
Local and global dynamics in Klebsiella pneumoniae outer mem- brane protein $A$ in lipid bilayers probed at atomic resolution
}

\author{
Olivier Saurel ${ }^{\star}$, I Iordan Iordanov, ${ }^{\mathrm{a} \dagger}$ Guillaume Nars, ${ }^{\mathrm{a}}$ Pascal Demange, ${ }^{\mathrm{a}}$ Tanguy Le Marchand, ${ }^{\mathrm{b}}$ Loren B. \\ Andreas, ${ }^{\mathrm{b}}$ Guido Pintacuda ${ }^{\mathrm{b}}$ and Alain Milon ${ }^{\star a}$ \\ a. Institut de Pharmacologie et de Biologie Structurale (CNRS/Université Paul Sabatier), Université de Toulouse, France \\ *E-mail: olivier.saurel@ipbs.fr, alain.milon@ipbs.fr. \\ b. Institut de Sciences Analytiques (UMR 5280 CNRS / ENS-Lyon / UCB Lyon 1), Université de Lyon, France
}

KEYWORDS: Extracellular loop, Klebsiella pneumoniae, MALDI-TOF mass spectrometry, Magic-angle spinning NMR, Protein dynamics

ABSTRACT: The role of membrane proteins in cellular mechanism strongly depends on their dynamics and solid-state MagicAngle Spinning (MAS) Nuclear Magnetic Resonance (NMR) is a unique method to exhaustively characterize motions of proteins in a lipid environment. Herein, we make use of advances in ${ }^{1} \mathrm{H}$-detected MAS NMR to describe the dynamics of the membrane domain of the Outer membrane protein A of Klebsiella pneumoniae (KpOmpA). By measuring ${ }^{1} \mathrm{H}-{ }^{15} \mathrm{~N}$ dipolar-coupling as well as ${ }^{15} \mathrm{~N} \mathrm{R}_{1}$ and $\mathrm{R}_{1 \rho}$ relaxation rates at fast $(60 \mathrm{kHz})$ MAS and high magnetic field $(1 \mathrm{GHz})$, we were able to describe the motions of the residues of the $\beta$-barrel as a collective rocking of low amplitude and of hundreds of nano-seconds time scale. Residual local motions at the edges of the strands, underscored by enhanced ${ }^{15} \mathrm{~N} \mathrm{R}_{1 \rho}$ relaxation rates, report on the mobility of the connected loops. In agreement with MAS NMR data, proteolysis experiments performed on the full length KpOmpA as well as on its membrane domain, reconstituted in liposomes or in detergent micelles, revealed in all cases the existence of a unique Trypsin cleavage site within the membrane domain (out of 16 potential Lys and Arg sites). This site is located in the extracellular loop L3, showing that it is highly accessible to protein-protein interactions. KpOmpA is involved in cell-cell recognition, for adhesion and immune response mechanisms. The $\mathrm{L} 3$ region may therefore play a key role in pathogenicity.

\section{INTRODUCTION}

Membrane proteins are key players in many cellular processes such as cell recognition or signal transmission. Their function is intimately connected to their capacity of adopting different conformations, and the characterization of the dynamical interconversion between states is therefore a major step for understanding their role in the cell. However, this very same inherent flexibility and the fluidity of their native lipid environment constitute a barrier for high-resolution X-ray determinations, and truncation of flexible loops, engineered mutants, or other stabilization strategies are often used to lock the protein into a conformation that will form large crystals.

Solid-state Magic-Angle Spinning (MAS) Nuclear Magnetic Resonance (NMR) is a technique capable of tackling structure of membrane proteins ${ }^{1-5}$ in lipid bilayers. Additionally, this technique has a unique potential to probe dynamics over a large range of timescales, ${ }^{6-8}$ providing important complementary insight for understanding function in these complex substrates. ${ }^{1-3,9-13}$

Over the last ten years, a thick portfolio of MAS NMR methods for accessing molecular dynamics has been devel- oped, and amplitude and timescale of complex motions can in principle be sampled along a protein chain. ${ }^{14-21}$ However, while remarkable site-specific studies of protein dynamics have been reported for few benchmark proteins, mainly in microcrystalline form, ${ }^{22-26}$ analogous systematic investigations are still rare for membrane proteins, mainly because of the lack of sensitivity and the difficulty to obtain the full assignment of the resonances in such samples. It is however essential to study membrane protein structure and dynamics in their fluid lipidic environment, in order to accurately describe topologies and protein-lipid interactions, as well as the specific dynamics of transmembrane domains and extracellular loops.

A first complete investigation was performed in a protein of 7 transmembrane helices reconstituted in lipids. ${ }^{27}$ Extensive measurements of dipole order parameters and ${ }^{15} \mathrm{~N} \mathrm{R}_{1 \rho}$ relaxation rates revealed that each individual secondary element undergoes sub-microsecond motions with a particular amplitude and timescale. This study clearly showed that collective dynamics is strongly connected to the protein topology. Membrane protein topology and dynamics may also be characterized in membrane-mimetic environments such as nano- 
discs $^{28}$ and oriented bicelles as was nicely illustrated by studies on cytochrome $\mathrm{P} 450$ and $\mathrm{b}(5)^{29,30}$.

Here we address the question of protein dynamics in fluid bilayers on the $\beta$-barrel trans-membrane domain of the outer membrane protein A from Klebsiella pneumoniae (KpOmpA). The transmembrane domain $\beta$-barrel was characterized by solution NMR (PDB code $2 \mathrm{~K} 0 \mathrm{~L}$ ), ${ }^{31}$ and is composed of $8 \beta$ strands, 4 long extracellular loops (L1-4) and 3 short periplasmic turns (T1-3). A flexible hinge region links the barrel with the soluble C-terminal domain of the molecule, which is responsible for binding to the peptidoglycan network in the bacterial periplasm. OmpA contributes to pathogenicity via its role in cell-cell adherence, its pro-inflammatory role and its implication in innate and adaptive immunity. This is usually facilitated by specific interaction between a cell surface protein on the bacterium and the extracellular matrix (ECM) proteins in the target cell, such as fibronectin. ${ }^{32,33}$ This suggests that the epitope for protein binding and cell adhesion, could be a flexible and well-presented extracellular loop. ${ }^{34}$

For the characterization of $\mathrm{N}-\mathrm{KpOmpA}$ reconstituted in a fluid lipid bilayer environment (proteoliposomes made of E.coli polar lipids) by solid-state NMR, we used ultra-high magnetic field and very fast MAS $(60 \mathrm{kHz})$ on a perdeuterated and amide-reprotonated sample. ${ }^{35,36}$ Under these conditions, ${ }^{1} \mathrm{H}$ detection allows measurement of well-resolved 2D solidstate NMR spectra of proteins with minimal signal overlap. ${ }^{37,38}$ These developments were shown not only to speed up backbone resonance assignment ${ }^{39-41}$ and structure determination, ${ }^{5,22,42,43}$ but also to provide sufficient sensitivity for the measurement of ${ }^{13} \mathrm{C}$ and ${ }^{15} \mathrm{~N}$ nuclear relaxation rates in microcrystalline and non-crystalline systems of biological interest. ${ }^{19,22,23,27,44-46}$ With the availability of even faster MAS rates, this approach can be extended to systems which are available in very small quantities or cannot be easily deuterated. ${ }^{13,24,43,47-51}$

The very fast MAS regime has an added advantage in relaxation studies, since the proton bath is well decoupled, alleviating potential interfering effects of coherent contributions. This opens the possibility for the site-specific measurement of $R_{1 p}$ relaxation parameters, which are intractable with fullyprotonated samples at slower MAS rates due to residual dipolar effects. ${ }^{17}$ Similarly, the measurement of ${ }^{1} \mathrm{H}^{-15} \mathrm{~N}$ dipolar couplings by REDOR and their derived order parameters becomes straightforward. ${ }^{52,53}$ Overall, this strategy enables a quantitative description of the dynamics, which in the case of membrane proteins is still challenging.

The analysis of solid-state NMR data reveals a combination of different types of motion in N-KpOmpA. In the lipid environment used in solid-state NMR or X-Ray diffraction experiments, we characterized a collective rocking of the barrel at sub-microsecond time scale. Slower residual local motions of the residues close to the loops were also detected with a particular emphasis at the extremity of the strand 6. This picture is supported by proteolysis experiments which indeed supports a different behaviour of the loop 3 connected to strand 6 and indicate the key-role played by this region for the interaction between the bacterial pathogen and the surface of the target cells.

\section{EXPERIMENTAL SECTION}

\section{Construction of the $\mathrm{KpOmpA}$ recombinant proteins}

The construction of the transmembrane domain of KpOmpA (N-KpOmpA, 210 a.a., $23.4 \mathrm{kDa}$ ) as a separate expressible product in a pET21c vector was described previously. ${ }^{31}$ The full-length polypeptide (F-KpOmpA, containing in addition the soluble Cterminal domain) was PCR-amplified from genomic DNA of Klebsiella pneumoniae strain 52145 (kindly provided by Dr. Tournebize, Institut Pasteur, Paris) using forward (GTGGCACTGCATATGAAAGCTACCGTAGCGCAGGCCGC TCCGAAAGATAAC) and reverse (GGTA CCAGCTCGAG AGCCGCCGGCTGAGTTACAACTTCT) primers, and Phusion Hot Start II High-Fidelity DNA Polymerase (Fynnzymes). The underlined sequences represent $\mathrm{NdeI}$ and XhoI restriction sites used for subcloning in pET26b vector. The cloned F-KpOmpA gene was sequence-verified (MilleGen). N-KpOmpA contains a short modification at the N-terminus (ARIMKAIFVLNA) and a hexahistidine tag at the C-terminus, immediately after the flexible hinge region of the molecule that natively links its two domains. F-KpOmpA (359 a.a., $38.7 \mathrm{kDa}$ ) contains a short modification at the N-terminus (MKATVAQA), the full-length polypeptide chain of KpOmpA and a thrombin cleavage site (LVPRGS) followed by a hexahistidine tag at the C-terminus. Both plasmids (pET21c-NKpOmpA and pET26b-F-KpOmpA) were electrotransferred to E.coli BL21 (DE3) strain.

\section{Protein expression and purification}

Both the $\mathrm{N}$ - and F-KpOmpA recombinant proteins lack the native N-terminal signal peptide for outer membrane localization and therefore accumulate as inclusion bodies in the cytoplasm. The expression/purification procedure for the triple isotopically labelled $\mathrm{N}$-terminal domain of the outer membrane protein from Klebsiella pneumoniae, [U- ${ }^{1} \mathrm{HN},{ }^{13} \mathrm{C},{ }^{15} \mathrm{~N},{ }^{2} \mathrm{H}$ ] N-KpOmpA, was described previously. ${ }^{31}$ Briefly, exponentially growing E. coli BL21 (DE3) cells harboring the pET26b-F-KpOmpA plasmid were induced with $1 \mathrm{mM}$ IPTG for 4 hours at $37^{\circ} \mathrm{C}$ and then collected. Upon cell lysis, the released inclusion bodies were washed and dissolved in $6 \mathrm{M}$ Guanidine/ $\mathrm{HCl}$ buffer containing $25 \mathrm{mM}$ Tris ( $\mathrm{pH} 8.5)$ and $5 \mathrm{mM}$ EDTA. The denatured protein was refolded in $15 x$ excess volume of $1 \%(w / v)$ Zwittergent 3-14 (Anatrace) buffer containing $25 \mathrm{mM}$ Tris ( $\mathrm{pH} 8.5$ ) and $150 \mathrm{mM} \mathrm{NaCl}$. The detergent concentration was then reduced to $0.1 \% \mathrm{ZW} 3-14$ via dialysis (12-14 kDa cutoff, Spectra/Por) and the protein was purified by affinity chromatography (Ni-NTA Superflow, QIAGEN) with elution step of $400 \mathrm{mM}$ imidazole. The imidazole was removed by a second dialysis and the final protein stock (in 0.1\% ZW3-14, 25 $\mathrm{mM}$ Tris ( $\mathrm{pH} \mathrm{8.5),} 150 \mathrm{mM} \mathrm{NaCl}$ ) was stored at $4^{\circ} \mathrm{C}$. The concentration of the purified proteins was determined spectrophotometrically by measuring the absorbance at $280 \mathrm{~nm}$ and using the theoretical molar extinction coefficients of $50880 \mathrm{M}^{-1} \mathrm{~cm}^{-1}$ and $56140 \mathrm{M}^{-1} \mathrm{~cm}^{-1}$ for $\mathrm{N}$ - and F-KpOmpA, respectively. The purity 
and successful refolding of the proteins was monitored with SDS PAGE.

\section{Proteoliposomes preparation}

Proteoliposomes containing N-KpOmpA were prepared as described previously, ${ }^{54}$ using detergent dilution method adapted from procedures described by Rigaud et al. ${ }^{55}$ Briefly, E. coli Polar Lipids Extract (PLE, Avanti Polar Lipids) was dissolved in chloroform, dried under nitrogen stream and then in a vacuum chamber overnight. This lipid film was dissolved at $10 \mathrm{mg} / \mathrm{ml}$ in $2 \% \mathrm{n}$ octyl- $\beta$-d-glucopyranoside (Carbosynth), $20 \mathrm{mM}$ Tris ( $\mathrm{pH} 8.5$ ), $100 \mathrm{mM} \mathrm{NaCl}$ and sonicated in a water bath several times. Purified $\mathrm{N}$ - or F-KpOmpA was bound to nickel-chelating resin, extensively washed with $2 \%$ n-octyl- $\beta$-d-glucopyranoside solution containing $20 \mathrm{mM}$ Tris ( $\mathrm{pH} 8.5$ ) and $100 \mathrm{mM} \mathrm{NaCl}$ and eluted with the same buffer supplemented with $400 \mathrm{mM}$ imidazole. Mixed micelles were formed by adding the E. coli Polar Lipids Extract solution to the protein eluate at the desired lipid-toprotein ratio (LPR), ranging from 0.5 to $5 \mathrm{w} / \mathrm{w}$ (equivalent to $\sim 15$ 150:1 $\mathrm{mol} / \mathrm{mol}$ ) in the different experiments, NMR samples were finally prepared at a LPR of $0.5 \mathrm{w} / \mathrm{w}$. This mixture was incubated for $10 \mathrm{~min}$ at $4{ }^{\circ} \mathrm{C}$ under gentle mixing and then dialyzed at $37^{\circ} \mathrm{C}$ against detergent-free buffer, until complete removal of the detergent and formation of large unilamellar vesicles. During the dialysis, the $\mathrm{NaCl}$ was gradually removed in $25-\mathrm{mM}$ steps. The final proteoliposomes sample is thus found in only $20 \mathrm{mM}$ Tris buffer ( $\mathrm{pH} 8.5)$.

\section{Proteolysis experiments}

The commercially available proteases 'Endoproteinase Lysine-C sequencing grade' (Lys-C, Roche) and 'Sequencing Grade Modified Trypsin' (Promega) were supplied as lyophilized powders and dissolved according to the manufacturer's instructions, resulting in $0.1 \mathrm{mg} / \mathrm{ml}$ stocks for each enzyme. Digestion experiments with Lys-C: for proteolysis experiments in 'complete digestion' conditions, $50-450 \mu \mathrm{g}$ of $\mathrm{N}-\mathrm{KpOmpA}(1-3 \mathrm{mg} / \mathrm{ml}$ in the $0.1 \% \mathrm{ZW} 3-14$ stock or in the proteoliposomes in $20 \mathrm{mM}$ Tris) were directly mixed with $10 \mu \mathrm{l}$ protease stock ( $1 \mu \mathrm{g}$ enzyme) to the desired substrate-to-enzyme ratio (SER, ranging from 50:1 to 450:1 w/w). The volumes ratio of this mixture was at least 10:1 in favor of the KpOmpA-buffer, in order to ensure that the reaction conditions are determined by the KpOmpA-buffer. The reaction was incubated at $37^{\circ} \mathrm{C}$ for several hours or overnight and then aliquots were taken for SDS PAGE and MALDI-TOF. The aliquot for mass spectrometry was added to equal volume of $0.1 \%$ trifluoroacetic acid (TFA) and $50 \%$ acetonitrile $(\mathrm{AcN})$ in $1: 1(\mathrm{v} / \mathrm{v})$ ratio. If the aliquot material was considered too diluted, it was dried under vacuum (miVac Duo Concentrator, GeneVac) and resuspended in $10 \mu \mathrm{l}$ of the same TFA/AcN solution. For monitoring the digestion kinetics of the protein, N- or F-KpOmpA (in micellar or bilayer environment) was treated in the same way, except that the reaction temperature was kept at $16^{\circ} \mathrm{C}$ for the micellar sample and at $37^{\circ} \mathrm{C}$ for the reconstituted protein sample. At certain time-points (0-120 $\mathrm{min})$, aliquots from the reaction mixture were collected and immediately added either to the SDS PAGE loading dye and boiled for $5 \mathrm{~min}$, or to equal volume of TFA/AcN solution and frozen at $-20^{\circ} \mathrm{C}$ until analysed by mass spectrometry.

\section{Mass spectrometry}

$1 \mu \mathrm{l}$ of each aliquot (1-2 $\mu \mathrm{g} \mathrm{KpOmpA}$ ) was deposited on a MALDI-plate and mixed with $1 \mu$ of matrix solution (either alpha-cyano-4-hydroxycinnamic acid (CHCA, Fluka Analytical) or 3,5-dimethoxy-4-hydroxycinnamic acid (SPA), as indicated in text). Upon drying at room temperature, the plate was loaded into the vacuum chamber of the spectrometer. All mass spectrometry measurements were performed on a Voyager DE-STR (Applied Biosystems) MALDI-TOF spectrometer, using Voyager Instrument Control Panel software for data acquisition and Data Explorer software for data processing and analysis. The spectra were acquired either in linear (1-50 kDa range, $25 \mathrm{kV}$ accelerating voltage, $300 \mathrm{~ns}$ delay time) or in reflectron (0.8-4 $\mathrm{kDa}$ range, 20 $\mathrm{kV}$ accelerating voltage, $240 \mathrm{~ns}$ delay time) mode, with positive ion detection and 1000 shots per spectrum in each case. Spectral calibration was done with commercial compounds of known sizes.

\section{NMR spectroscopy}

All experiments were carried out on Bruker Avance III 1000 $\mathrm{MHz}$ and $800 \mathrm{MHz}$ standard bore spectrometers (operating at static fields of 23.4 and $18.7 \mathrm{~T}$, respectively), both equipped with a $1.3 \mathrm{~mm}$ triple tuned $\left({ }^{1} \mathrm{H},{ }^{13} \mathrm{C},{ }^{15} \mathrm{~N}\right) \mathrm{CP}$-MAS probe, at $\omega \mathrm{r} / 2 \pi=60$ $\mathrm{kHz}$ sample spinning rate. Details on experimental setup and analysis of NMR experiments are given as Supporting Information. Proton detected NMR spectra were acquired as detailed in $^{39}$, namely, 2D CP and J-based HN correlation, 3D (H)CANH, $(\mathrm{H}) \mathrm{CONH}$ and $(\mathrm{H})(\mathrm{CA}) \mathrm{CB}(\mathrm{CACO}) \mathrm{NH}$ spectra. ${ }^{15} \mathrm{~N} \mathrm{~T}_{1}$ and $\mathrm{T}_{1}$ measurements were performed by the insertion of a $\mathrm{T}_{1}$ relaxation delay and a ${ }^{15} \mathrm{~N}$ spin-lock pulse respectively just after the ${ }^{1} \mathrm{H}$ to ${ }^{15} \mathrm{~N}$ $\mathrm{CP}$ in the $\mathrm{HN}$ correlation spectrum. $\mathrm{T}_{1}$ relaxation delays were set to $1,2,3,5,25$ and $50 \mathrm{~s}$ while ${ }^{15} \mathrm{~N}$ spin lock pulse durations were set to $0.5,10,15,20,40,60$ and $100 \mathrm{~ms}$. For the measurement of the averaged ${ }^{15} \mathrm{~N}-{ }^{1} \mathrm{H}$ dipolar coupling, a REDOR block ${ }^{56}$ was inserted after tl evolution; this block consisted of $3.4 \mu$ s ${ }^{1} \mathrm{H} \pi-$ pulses applied each half rotor period, leading to a dephasing due to heteronuclear dipolar recoupling. Twelve spectra were acquired with the following dephasing times 67, 100, 133, 167, 200, 233, 267, 300, 333, 367, 400 and $433 \mu$ s. Chemical shifts were referenced to DSS. The sample temperature was $15^{\circ} \mathrm{C}$, as indicated by the chemical shift of supernatant water. For the fitting of the Gaussian axial fluctuation (GAF) dynamics model to experimental data, for each site $\langle\mathrm{S}\rangle,{ }^{15} \mathrm{~N} \mathrm{R}_{1}$ and ${ }^{15} \mathrm{~N} \mathrm{R}_{1 \rho}$ were calculated according to well-known formulae for dipolar and CSA relaxation, reproduced in the SI. ${ }^{14,15}$

\section{RESULTS AND DISCUSSION}

Figure 1A shows the dipolar-based ${ }^{15} \mathrm{~N}-{ }^{1} \mathrm{H}$ correlation spectrum of [U- $\left.{ }^{1} \mathrm{HN},{ }^{2} \mathrm{H},{ }^{13} \mathrm{C},{ }^{15} \mathrm{~N}\right]$-labelled KpOmpA in lipid bilayers, which features correlations originating from the most rigid part of the protein. The peaks are spread in the direct ${ }^{1} \mathrm{H}$ dimension from 8 to $10 \mathrm{ppm}$, a dispersion indicative of secondary structure elements, here a $\beta$-barrel. In order to assign the resonances, the solution chemical shifts ${ }^{31}$ were used along with three 3D spectra, namely $(\mathrm{H}) \mathrm{CANH},(\mathrm{H}) \mathrm{CONH}$ and $(\mathrm{H})(\mathrm{CA}) \mathrm{CB}(\mathrm{CACO}) \mathrm{NH}$, that correlate $\mathrm{CA}_{\mathrm{i}} \mathrm{N}_{\mathrm{i}} \mathrm{H}_{\mathrm{i}}, \mathrm{CO}_{\mathrm{i}-1} \mathrm{~N}_{\mathrm{i}} \mathrm{H}_{\mathrm{i}}$ and $\mathrm{CB}_{\mathrm{i}-1} \mathrm{~N}_{\mathrm{i}} \mathrm{H}_{\mathrm{i}}$ nuclei respectively. ${ }^{39}$ Joint evaluation of the three 
spectra allowed residue typing and spin-system $\left(\mathrm{CB}_{\mathrm{i}-1}, \mathrm{CO}_{\mathrm{i}-1}\right.$, $\mathrm{N}_{\mathrm{i}}, \mathrm{H}_{\mathrm{i}} \mathrm{CA}_{\mathrm{i}}$ ) identification, which could reliably be compared to the solution data resulting in unambiguous assignment of 49 residues (Fig. 1A and Table S1). The NMR pulse sequences used for assignment contain multiple cross polarization steps and therefore are a strong filter which selects only the more rigid portions of the protein. It is not surprising that all but one of the assigned residues (e.g; residues colored in green in Figure 1C) are found throughout the $\beta$-barrel core of the protein. It is noteworthy that the intensity of these dipolar correlations represents a first reporter of local flexibility, with the signals becoming weaker at the edge of $\beta$-strands. As seen on figure $1 \mathrm{~B}$, this feature is particularly obvious considering the peak intensity of two isolated glycines located at the top of strand 1 and 2 (e.g G26 and G53 and shown to be in chemical exchange in a micelle environment ${ }^{31,54}$ ) compared to G22, G58 and G71 in the middle of the $\beta$-strands 1, 2 and 3 respectively. Interestingly, no extracellular loops are detected in this approach, and neither are the first nine residues in $\beta$-strand 6 flanking L3 (Figure 1C). This suggests transient unfolding of this portion of the $\beta$-strand, or the presence of a topology in lipids different from that found in detergent micelles. In order to further investigate residues from the extracellular loops, ${ }^{1} \mathrm{H}$ ${ }^{15} \mathrm{~N}$ correlation based on INEPT transfer schemes were measured as well. However, they failed to reveal distinct cross peaks arising from loops L1 to L4, showing that none of these loop is in a truly random coil conformation with fast, sub-ns motion. To investigate dynamics in the $\beta$-barrel core, we first measured the amide ${ }^{15} \mathrm{~N}-{ }^{1} \mathrm{H}$ dipolar coupling ${ }^{52}$ on every assigned peak (Figure 2B,D). The order parameter $\langle\mathrm{S}\rangle$, defined as the fraction of the rigid limit coupling, is sensitive to any motional process faster than the ${ }^{15} \mathrm{~N}-{ }^{1} \mathrm{H}$ dipolar coupling $(\approx 11 \mathrm{kHz})$. In this measurement, $\langle\mathrm{S}\rangle$ is fairly homogeneous along the $\beta$ barrel (Figure $2 \mathrm{~B}$ and Table S2), with all residues displaying a value above 0.8 (average value: $0.88 \pm 0.05$ ). This indicates that there is only moderate-amplitude motion throughout the assigned portion of N-KpOmpA and that these residues within the barrel display similar motion. Amides for which there were not observable ${ }^{15} \mathrm{~N}-{ }^{1} \mathrm{H}$ cross peaks indicate more substantial local motion, which occurred for residues 102-111 in turn 2, the periplasmic side of strand 5 and residues 138-146 in the extracellular side of strand 6. The consistently high values of the order parameter along the sequence also demonstrates the absence of fast axial diffusion (Figure 2A,B), which under our experimental conditions would impose a modulation of $\langle\mathrm{S}\rangle$ dependent on the angle between the amide bond vector and the axis of rotation illustrated by the bars in Figure 2B. As discussed in ref. ${ }^{57}$, axial diffusion would lead to a drastic reduction of the resolution which is not observed in our experimental conditions, although the measurements were performed above the lipid phase transition temperature. This might be a consequence of the low lipid to protein ratio 0.5 $(\mathrm{w} / \mathrm{w})$, which results in a membrane with greater viscosity and a reduced diffusion rate.
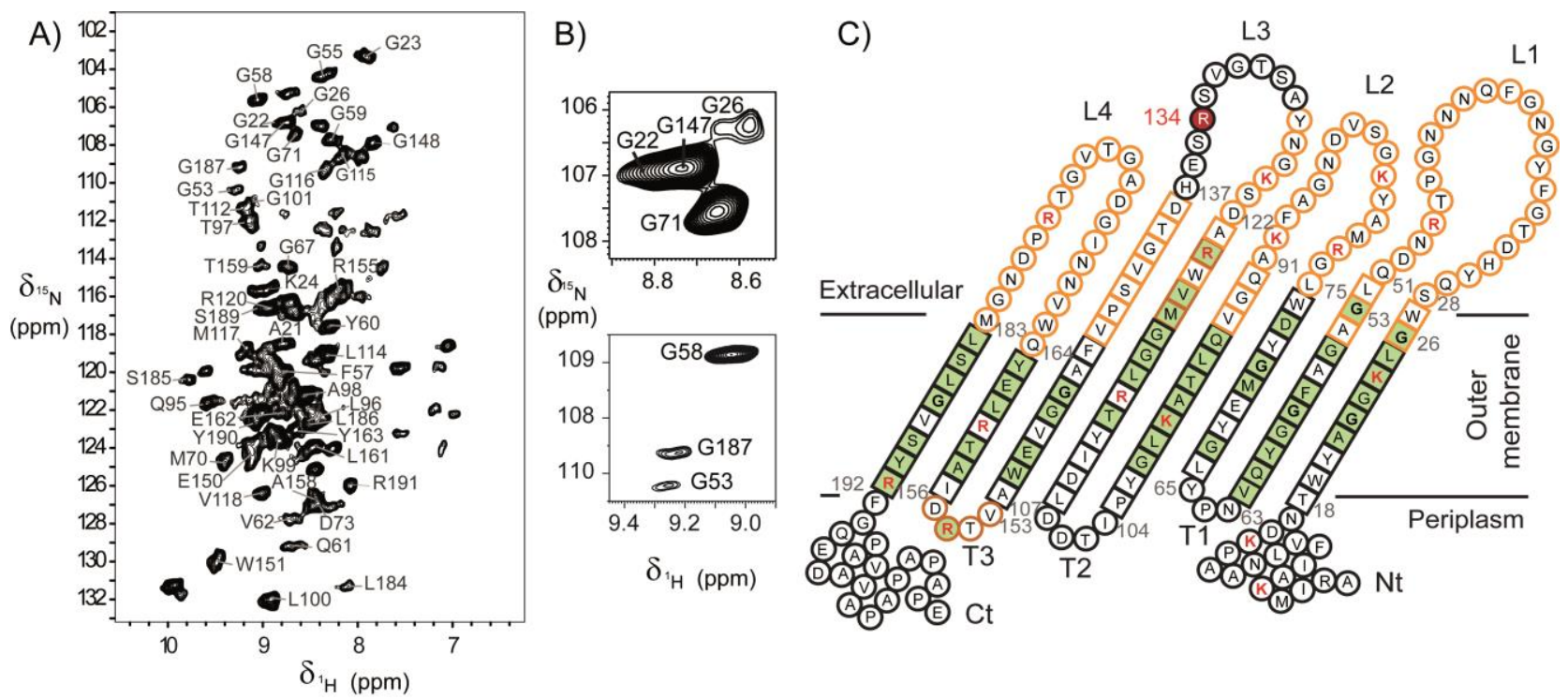

Figure 1. (A) Very fast MAS ${ }^{15} \mathrm{~N}-{ }^{1} \mathrm{H}$ correlation spectrum of $\left[\mathrm{U}-{ }^{1} \mathrm{HN},{ }^{2} \mathrm{H},{ }^{13} \mathrm{C},{ }^{15} \mathrm{~N}\right]$-labelled N-KpOmpA reconstituted in E. Coli polar extract liposomes, recorded at $1 \mathrm{GHz}$ spectrometer and $60 \mathrm{kHz}$ MAS frequency and $15^{\circ} \mathrm{C}$. Assigned residues are annotated. (B) Two regions of (A) containing glycine resonances. Glycine residues at the strand-to-loop transition (i.e., G26 and G53) are weaker compared to G22 and G58 placed in the same strand and G71 in $\beta$-strand 3. (C) Topological representation of assigned residues in N-KpOmpA illustrating residue-specific mobility within distinct protein segments and Arg and Lys potential cleavage sites for Trypsin. Residues within $\beta$-sheet and random coil regions are represented by squares and circles, respectively. Color code: assigned residues are highlighted in green, residues 
that exhibit intermediate time scale motions are colored in orange, Arg and Lys residues are colored in red and the unique cleavage site (i.e., R134) accessible to Trypsin is highlighted and annotated in red while the surrounding residues within loop 3 are in black.

(A)

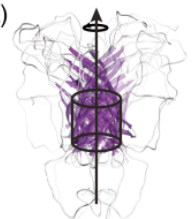

(C)

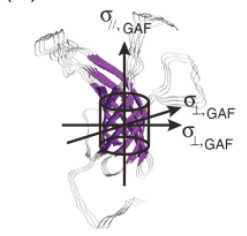

(E)

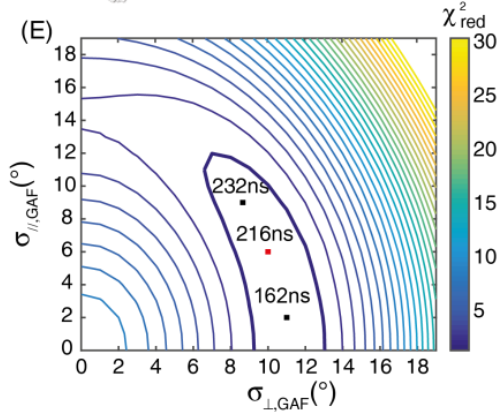

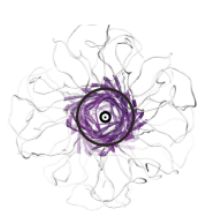

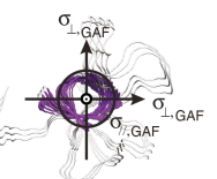

\section{(F)}

(B)
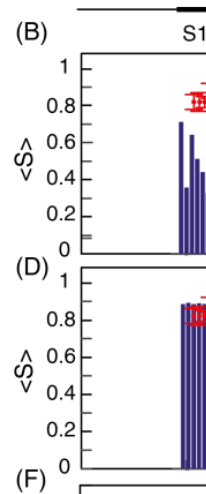

L1
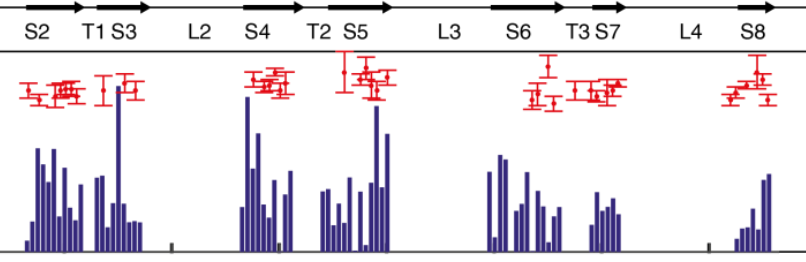

is 30
$\simeq^{2} 20$
10

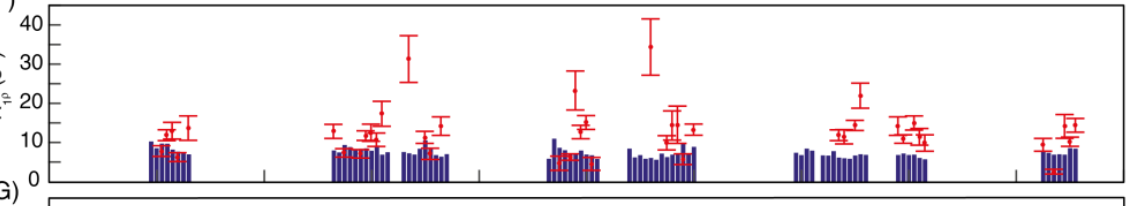

(G)
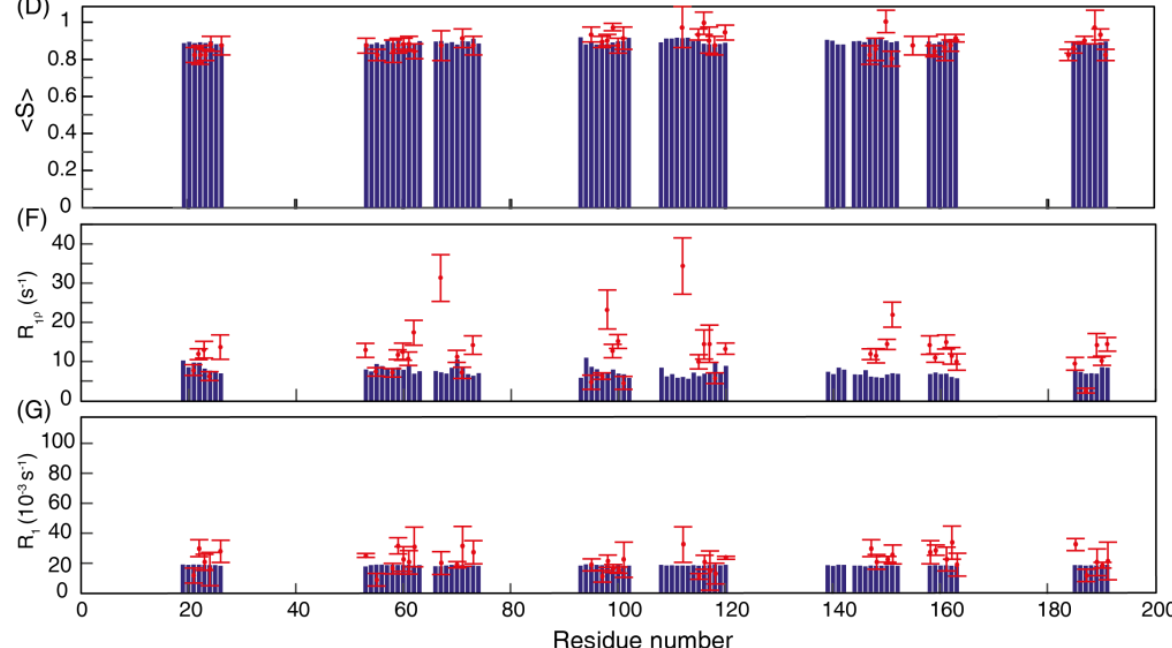
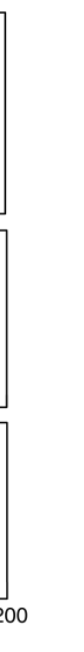

Figure 2. Residue-specific characterization of dynamics in $\left[\mathrm{U}-{ }^{1} \mathrm{HN},{ }^{2} \mathrm{H},{ }^{13} \mathrm{C},{ }^{15} \mathrm{~N}\right]$-labelled KpOmpA reconstituted in E. coli polar extract liposomes with a lipid to protein ratio of $0.5(\mathrm{w} / \mathrm{w})$ and at $25^{\circ} \mathrm{C}$. Representation of $(\mathrm{A})$ a full rotation of $\mathrm{kp}-\mathrm{OmpA}$ around the cylinder axis due to axial diffusion along the bilayer normal and $(\mathrm{C})$ of rocking motion with the amplitudes $\sigma_{/ /}=6^{\circ}$ and $\sigma_{\perp}=10^{\circ}$. (B,D) ${ }^{15} \mathrm{~N}-{ }^{1} \mathrm{H}$ bond order parameters $\langle\mathrm{S}>$ as measured by REDOR (red dots) and calculated for the full rotation (B, blue bars) and the rocking motion (D, blue bars). (E) Contour plot of the reduced chi square function $\left(\chi^{2}\right.$ red $)$ calculated as a function of the amplitudes of the rocking motion. The dots correspond to different pairs of amplitudes used to calculate ${ }^{15} \mathrm{~N} \mathrm{R}_{1}$ and $\mathrm{R}_{1 \rho}$ relaxation rates. Above them are depicted the correlation time ( $\tau_{\mathrm{GAF}}$ ) obtained from the fits. The value of $\chi^{2}$ red corresponding to the thicker contour line is 2 . (F) and (G), respectively, ${ }^{15} \mathrm{~N} R_{1 \rho}$ and $R_{1}$ relaxation rates determined experimentally (red dots) and calculated with $\sigma_{/ /}=6^{\circ}, \sigma_{\perp}=10^{\circ}$ and $\tau_{\mathrm{GAF}}=216 \mathrm{~ns}$ (blue bars). Residues for which the agreement is poor (G67, A98, T112, W151) are subject to local motion.

An explanation of the experimental values compatible with the low fluidity of the lipid bilayer is provided by rocking motion of the transmembrane domain. We modelled such anisotropic dynamical process of the $\beta$-barrel with the help of a Gaussian axial fluctuation (GAF) formalism. ${ }^{58,59}$ In this approach, the amide- $\mathrm{NH}$ bonds fluctuate around three axes corresponding to the axes of inertia of the protein. The corresponding order parameters depend on the angle between each amide bond vector and the molecular frame, and on the fluctuation amplitudes $\left(\sigma_{\alpha}, \sigma_{\beta}, \sigma_{\gamma}\right)$ of the collective motions (Figure $2 \mathrm{C}$, details in the SI). Figure $2 \mathrm{D}$ shows that a set of small amplitudes with cylindrical symmetry of the barrel $\left(\sigma_{\alpha}=\sigma_{/ / \sim} \sim 6^{\circ}\right.$, $\left.\sigma_{\beta}=\sigma_{\gamma}=\sigma_{\perp} \sim 10^{\circ}\right)$ reproduces accurately the experimental order parameter profile. Using this model, a small range of amplitudes $\left(\sigma_{/ /}\right.$and $\left.\sigma_{\perp}\right)$ describes equally well the experimental values. This is depicted in Figure 2E, which shows the agreement between experimental and back-calculated order parameters with respect to the two individual amplitudes, expressed by the reduced chi square function $\left(\chi^{2}\right.$ red $)$. The best agreement is reached for $\sigma_{/ /}$ranging between $\sim 0^{\circ}$ and $\sim 12^{\circ}$ and $\sigma_{\perp}$ between $\sim 9^{\circ}$ and $\sim 13^{\circ}$. This plot strikingly shows that the two amplitudes of fluctuation are anti-correlated: relatively high values of $\sigma_{/ /}\left(\sim 11^{\circ}\right)$ together with smaller values of $\sigma_{\perp}\left(\sim 8^{\circ}\right)$ explain the experimental order parameters similarly well than low values of $\sigma_{/ /}\left(\sim 0^{\circ}\right)$ and higher values of $\sigma_{\perp}\left(\sim 11^{\circ}\right)$.

A deeper insight into this motional process is provided by ${ }^{15} \mathrm{~N}$ relaxation rates, which are sensitive both to amplitude and timescales of motions. ${ }^{15} \mathrm{~N} \mathrm{R}_{1}{ }^{14}$ and $\mathrm{R}_{1 \rho}{ }^{16,17}$ relaxation rates were measured for all the resolved amide signals, and are reported in Figure $2 \mathrm{~F}$ and $2 \mathrm{G}$ (and Table $\mathrm{S} 2$ ). ${ }^{15} \mathrm{~N} \mathrm{R}_{1}$ relaxation rates range from 0.008 to $0.033 \mathrm{~s}^{-1}$ (average value of $0.022 \mathrm{~s}^{-1}$ ) and are relatively constant for the observed peaks, while ${ }^{15} \mathrm{~N} \mathrm{R}_{1 \rho}$ relaxation rates range from 2 to $34 \mathrm{~s}^{-1}$ (average value of $12 \mathrm{~s}^{-1}$ ). ${ }^{15} \mathrm{~N} R_{1 \rho}$ relaxation rates are particularly sensitive to $\mathrm{ms}-\mu \mathrm{s}$ motions and exchange phenomena. Most ${ }^{15} \mathrm{~N} R_{1 \rho}$ rates are clustered around 7 to $15 \mathrm{~s}^{-1}$ with a few exceptions. Rates higher 
than $20 \mathrm{~s}^{-1}$ are found for residues G67, A98, T112 and W151, mostly located in turns or close to the strand extremities and indicate the existence of slow internal motions. ${ }^{27}$ Within the sensitivity and resolution of these experiments, $\mathrm{R}_{1 \rho}$ could only be measured for residues displaying a moderate increase in $R_{1 \rho}$, while stronger increases lead to peak disappearance, as was observed for the extracellular half of strand 6 .

This evidence thus supports the hypothesis that the $\mathrm{N}$ terminal half of $\beta$-strand 6 samples a variety of conformations beyond that determined in detergent micelles. It is also interesting to compare the average $R_{1 \rho}$ value with values from the recent literature. Ladizhansky and co-workers studied a seven helices transmembrane protein, sensory rhodopsin, in proteoliposomes made of DMPC and DMPA, at $280 \mathrm{~K}$, i.e. 15 degrees below the lipid phase transition temperature, the lipids being in gel phase. ${ }^{27}$ The $R_{1 \rho}$ of residues in the rigid part of the protein ranged from 1 to $5 \mathrm{~s}^{-1}$, and residues with $R_{1 \rho}$ between 10 to $30 \mathrm{~s}^{-1}$ were identified as subject to slower internal motions, in the $100 \mathrm{~ns}$ time scale. Another interesting point of comparison is given by the work of Schanda and co-workers who studied ubiquitin relaxation rates in several crystal forms.$^{26}$ In a rigid crystal, most $R_{1 \rho}$ values ranged from 3 to $6 \mathrm{~s}$ 1, while in a more hydrated crystal they ranged from 7 to $17 \mathrm{~s}^{-1}$ (that is very similar to what observed here). These higher relaxation rates were attributed to a rocking motion of ubiquitin within the most hydrated crystal form, with a time scale in the 0.1 to $1 \mu$ s range. A combined analysis of order parameters and relaxation rates allows a precise characterisation of the rocking motion of KpOmpA. Within the GAF model, once the fluctuation amplitudes are defined by the order parameter, ${ }^{15} \mathrm{~N}$ $R_{1}$ and $R_{1 \rho}$ relaxation rates depend solely on the timescales $\left(\tau_{/ /}\right.$ and $\tau_{\perp}$ ) of the motion. These parameters can therefore be extracted by fitting the experimental rates to the ones calculated according to the expressions for dipolar and CSA relaxation. Within the approximation $\tau_{\mathrm{GAF}}=\tau_{/ /}=\tau_{\perp}$, this procedure was performed for a pair of amplitudes (red square in Figure $2 \mathrm{E}$ ), and the result of the fit is depicted in Figure 2F and 2G. Comparable results are obtained for different sets of amplitudes (black squares in Figure 2E), and are reported in Figure S4. The correlation times $\tau_{\mathrm{GAF}}$ obtained are about $200 \mathrm{~ns}$. These timescales found for KpOmpA are close to the timescale of rocking motion of ubiquitin in hydrated crystals, explaining the similarity of the ${ }^{15} \mathrm{~N}$ relaxation rates between the two studies. As expected for multiaxial fluctuations, both ${ }^{15} \mathrm{~N} \mathrm{R}_{1}$ and $\mathrm{R}_{1 \rho}$ profiles are rather flat. Residues R155, W151, A98, G67, T112, are thought to possess internal motion and therefore have relaxation rates higher than calculated in the case of pure rocking motion. Overall, a fairly detailed dynamics picture of KpOmpA (Figure 1C) results from these data when considered along with previously published observations. ${ }^{31,54}$ The four loops are subject to ms- $\mu$ s motions, being observable neither via CP based transfer nor via INEPT based transfer under MAS conditions. Dynamics of the $\beta$-barrel are precisely described as collective rocking motions thanks to a GAF model.
Small amplitude fluctuations $\left(\sim 10^{\circ}\right)$ occurring at hundreds of nanoseconds explain the order parameters obtained experimentally, as well as most of the relaxation rates. The $\beta$-barrel is homogeneously rigid within strands $1-4$ and $7-8$, while the periplasmic side of strand 5 and the extracellular side of strand 6 display conformational disorder which leads to peak suppressions in the ${ }^{15} \mathrm{~N}-{ }^{1} \mathrm{H} 2 \mathrm{D}$ correlations. The observed motion of these strands highlights increased disorder in loop 3 compared to the other loops, which is corroborated by the proteolysis experiments detailed below.

Since the extracellular loops residues were not detected by ${ }^{1} \mathrm{H}$ solid-state NMR, we resorted to a completely orthogonal approach, based on proteolysis experiments, a widely used tool in structural biology of membrane proteins. ${ }^{60,61}$ It has long been recognized that loop mobility and accessibility is associated with efficient proteolysis. ${ }^{62-65} \mathrm{KpOmpA}$ contains 18 Lys residues ( 7 in the $\mathrm{N}$-terminal and 11 in the C-terminal domains) and 15 Arg residues ( 9 in the N-terminal and 6 in the C-terminal domains), thus potentially 33 cleavage sites for Trypsin, 16 of which are in the N-terminal domain, Fig. 1C). Considering the very large number of cleavage sites we first used Lys-C, a protease specific for lysine residues. Surprisingly, the N-terminal domain proved to be fully resistant to proteolysis, both when solubilized in detergent micelles or reconstituted in lipid bilayers composed of polar lipid extract (PLE) from E. coli (Fig. S1 and S2), while the C-terminal domain was fully digested (e.g. $50 \%$ in proteoliposomes due to the two possible protein orientations).

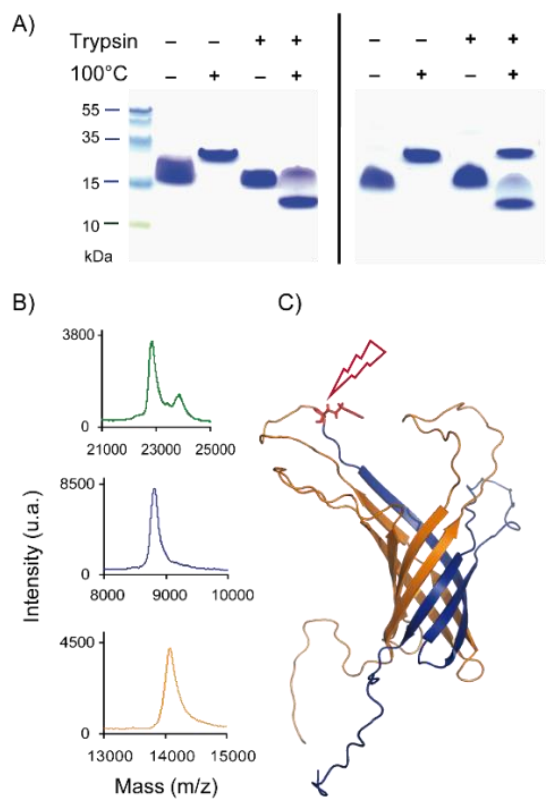

Figure 3. Complete digestion of N-KpOmpA with Trypsin: (A) SDS PAGE of digested N-KpOmpA in left panel: solubilized in 0.1 \% ZW3-14 micelle (SER $=200: 1 \mathrm{w} / \mathrm{w}, 37^{\circ} \mathrm{C}$ for $2.5 \mathrm{~h}$ ) or in right panel: reconstituted in PLE liposomes $(\mathrm{SER}=100: 1 \mathrm{w} / \mathrm{w}, \mathrm{LPR}=$ $60 \mathrm{~mol} / \mathrm{mol}, 37^{\circ} \mathrm{C}$ for $4 \mathrm{~h}$ ). The presence or the absence of Trypsin and of heat denaturation $\left(100^{\circ} \mathrm{C}\right)$ of the aliquots are indicated respectively with ' + ' and '-' above each well. (B) Normalized 
MALDI-TOF linear mode spectra of digested N-KpOmpA in PLE liposomes, showing intact N-terminal domain at $\sim 22.8 \mathrm{kDa}$ (green) and the cleaved fragments at $\sim 8.8 \mathrm{kDa}$ (blue) and $\sim 14.1$ $\mathrm{kDa}$ (orange). (C) 3D structures of the $\mathrm{N}$-terminal domains (PDB code $2 \mathrm{~K} 0 \mathrm{~L}$ ) with a color coding corresponding to the above fragments.

The proteolysis resistance was observed for up to $12 \mathrm{~h}$ at 37 ${ }^{\circ} \mathrm{C}$ and at a substrate to enzyme ratio (SER) of up to 100 in ZW3-14 detergent micelles as well as in E. coli proteoliposomes at a lipid to protein molar ratio of 15,60 and 150 and in nine different $20 \mathrm{mM}$ Tris buffer conditions ( $\mathrm{pH} \mathrm{6,7}$ and 8; $[\mathrm{NaCl}]=0,150$ and $300 \mathrm{mM}$ ). As seen in Figure 1C, three Lys cleavage sites in the extracellular loops are located in loops L2 and the beginning of loop L3 and thus do not report on L1 and L4 loop accessibility. Therefore, we used Trypsin capable of cleavage at both Lys and Arg residues, thus covering positions in all four loops. Figure 3 shows that heat denaturation of the Trypsin-digested N-KpOmpA (in 0.1\% ZW3-14 micelles and in proteoliposomes) resulted in fragments of the $\beta$-barrel. MALDI-TOF analysis (Figure 3B) identified the cleavage products as fragments of sizes $14057 \pm 10$ and $8814 \pm 9 \mathrm{Da}$ (in the micellar sample), and $14083 \pm 10$ and $8805 \pm 9 \mathrm{Da}$ (in the PLE sample) closely matching the calculated sizes of the fragments generated upon cleavage at Arg134 on loop L3. Thus, over the 16 potential cleavage sites, a unique reaction occurred at Arg134, whatever the amount of protease or the incubation time tested. This was observed both on the N-terminal domain and on the full-length protein, in micelles and in lipid bilayers. The $\beta$-barrel (containing 2 Lys and 5 Arg) was expected to be protected from proteolysis. The unique cleavage site at Arg134 was more surprising given 7 potential sites in extracellular loops, one Arg in L1, L2, L3 and L4, two Lys in L2 and one Lys at the beginning of L3 (Figure 1C). Considering that proteolytic cleavage is limited by loop motion and accessibility, ${ }^{62,63}$ this points to particular mobility of loop L3 in its C-terminal half (since Lys 124 at the beginning of loop L3 is not cleaved).

These results indicate that the L3 loop may be a key region of KpOmpA responsible for the adherence of the bacterial pathogen to the surface of the target cells, via its unique capacity to adapt its shape to target cells surface receptors. This result appears to be general across a variety of organisms based on sequence alignment (Table S3). We found that pathogenic strains of Enterobacteriaceae (Klebsiella, Salmonella, Shigella, Yersinia, Citrobacter) appear to have longer L3 loops, while a short L3 loop is present in non-pathogenic strains, such as Enterobacter (the situation in E. coli strains is more complex in this respect $\left.^{66}\right)$. It has been shown that OmpA from Mannheimia haemolytica A1 (a bacterium associated with bovine pneumonic pasteurellosis) is involved in fibronectin binding which is abolished by treatment of the bacterium with Tryp$\sin .{ }^{34} M$. haemolytica possesses a long L3 loop, with two potential sites for Trypsin (VQQHVDKDSRIKV compared to GNYASTGVSRSE for $K$. pneumoniae and the shorter
NVYGKN for E. coli). It is thus tempting to speculate that the L3 loop is highly flexible and well presented to the outer cell surface, contrary to the other extracellular loops, and thus accessible to Trypsin. This makes it an important epitope for protein binding and cell adhesion.

\section{CONCLUSIONS}

In conclusion, application of state-of-the-art ${ }^{1} \mathrm{H}$ detected techniques using ultra-fast magic-angle spinning and ultrahigh magnetic fields allow site-specific quantification of the kpOmpA barrel dynamics in lipid membranes. The measurement of ${ }^{1} \mathrm{H}_{-}{ }^{15} \mathrm{~N}$ dipolar couplings by REDOR indicates the presence of relatively uniform motion along the primary sequence. Collective rocking of the $\beta$-barrel with small amplitude $\left(\sim 10^{\circ}\right)$ is sufficient to explain the data. Measurement of scaled dipolar couplings reports on motions faster than the rigid limit frequency but not the time scales of motions. For this, we measured ${ }^{15} \mathrm{~N}_{1}$ and $R_{1 \rho}$ relaxation rates and found that the rocking model can explain the relaxation rates with a time scale of hundreds of nanoseconds. However, some residues close to the loops showed particularly enhanced $R_{1 \rho}$, indicating slower local motion related to the dynamics of the loops. In particular, residues at the extremity of strand 6 connected to loop 3 were assumed to transiently unfold as they are not detected with our approach. This finding is supported by trypsin cleavage experiments where a specific feature of the L3 loop of KpOmpA was identified, namely motion of sufficient amplitude for productive contacts with Trypsin.

In the conditions used for solid-state NMR, the dense protein packing constrains the motion of the barrel. The resulting motion is therefore a collective rocking with amplitudes of around ten degrees and a timescale of hundreds of nanoseconds. This may represent a general phenomenon in samples studied by other physical methods such as X-Ray crystallography. It is therefore necessary to take this type of motion into account to interpret local dynamics. Here, the residual dynamics of the residues at the edges of the barrel was informative about the motions of the loops. Combination of solid-state NMR with proteolysis experiment highlights the specific dynamics of the L3 loop which by extension is expected to be particularly accessible for interactions with other proteins required for cell-cell adhesion mechanisms.

Supporting Information. Detailed procedures for each method, theory of NMR data analyses, complementary results and data. This material is available free of charge via the Internet at http://pubs.acs.org.

\section{AUTHOR INFORMATION}

\section{Corresponding Authors}

* Institut de Pharmacologie et de Biologie Structurale, Université de Toulouse, CNRS, UPS, France

E-mail: olivier.saurel@ipbs.fr, alain.milon@ipbs.fr 


\section{Present Address}

$\uparrow$ Semmelweis University, Department of Medical Biochemistry, Budapest, Hungary.

\section{Author Contributions}

The manuscript was written through contributions of all authors. / All authors have given approval to the final version of the manuscript.

\section{ACKNOWLEDGMENT}

We acknowledge financial support from PICT - Genotoul platform of Toulouse, CNRS, Université de Toulouse-UPS, European structural funds, the Midi-Pyrénées region, CNRS (IR-RMN FR3050) and from the European Research Council (ERC) under the European Union's Horizon 2020 research and innovation programme (ERC-2015-CoG GA n ${ }^{\circ} 648974$ ). II was funded by an EU Initial Training network (FP7/2007-2013, grant agreement no. 211800). LBA was supported by a MC incoming fellowships (REA grant agreements n624918 "MEM-MAS”). We thank Dr. Lewandowski for providing a Matlab code for simulating ${ }^{15} \mathrm{~N} \mathrm{R}_{1}$ values via the website http://www.ens-lyon.fr/crmn/crmn/ index.html

\section{ABBREVIATIONS}

KpOmpA, outer membrane protein A from Klebsiella pneumonia; F-KpOmpA the full length protein; N-KpOmpA, its transmembrane domain; IPTG, isopropyl $\beta$-D-1-thiogalactopyranoside; ZW3-14, Zwittergent 3-14 (n-tetradecyl-N,N-dimethyl-3ammonio-1-propanesulfonate); PLE, E. coli Polar Lipids Extract; Lys-C, Lysine-C endoprotease; MALDI-TOF, matrix-assisted laser desorption/ ionization - time-of-flight mass spectrometry; LPR, lipid-to-protein ratio; SER, substrate-to-enzyme ratio; TFA, 2,2,2trifluoroacetic acid; AcN, acetonitrile; CHCA, alpha-cyano-4hydroxycinnamic acid; SPA, sinapinic acid (3.5-dimethoxy-4hydroxycinnamic acid)

\section{REFERENCES}

(1) Etzkorn, M.; Martell, S.; Andronesi, O. C.; Seidel, K.; Engelhard, M.; Baldus, M. Angew. Chem. Int. Ed. Engl. 2007, 46, 459.

(2) Shi, L. C.; Lake, E. M. R.; Ahmed, M. A. M.; Brown, L. S.; Ladizhansky, V. Biochim. Biophys. Acta 2009, 1788, 2563.

(3) McDermott, A. Annu. Rev. Biophys. 2009, 38, 385.

(4) Cady, S. D.; Schmidt-Rohr, K.; Wang, J.; Soto, C. S.; DeGrado, W. F.; Hong, M. Nature 2010, 463, 689.

(5) Andreas, L. B.; Reese, M.; Eddy, M. T.; Gelev, V.; Ni, Q. Z.; Miller, E. A.; Emsley, L.; Pintacuda, G.; Chou, J. J.; Griffin, R. G. J. Am. Chem. Soc. 2015, 137, 14877.

(6) Lewandowski, J. R. Acc. Chem. Res. 2013, 46, 2018.

(7) Krushelnitsky, A.; Reichert, D.; Saalwächter, K. Acc. Chem. Res. 2013, 46, 2028.

(8) Schanda, P.; Ernst, M. Prog. Nucl. Magn. Reson. Spectrosc. 2016, 96,1 .

(9) Cady, S. D.; Goodman, C.; Tatko, C. D.; DeGrado, W. F.; Hong, M. J. Am. Chem. Soc. 2007, 129, 5719.

(10) Bhate, M. P.; Wylie, B. J.; Tian, L.; McDermott, A. E. J. Mol. Biol. 2010, 401, 155.

(11) Struts, A. V.; Salgado, G. F. J.; Brown, M. F. Proc. Natl. Acad. Sci. USA 2011, 108, 8263.
(12) Park, S. H.; Casagrande, F.; Das, B. B.; Albrecht, L.; Chu, M.; Opella, S. J. Biochemistry 2011, 50, 2371.

(13) Yang, J.; Aslimovska, L.; Glaubitz, C. J. Am. Chem. Soc. 2011, 133, 4874.

(14) Giraud, N.; Blackledge, M.; Goldman, M.; Bockmann, A.; Lesage, A.; Penin, F.; Emsley, L. J. Am. Chem. Soc. 2005, 127, 18190.

(15) Lewandowski, J. R.; Sein, J.; Sass, H. J.; Grzesiek, S.; Blackledge, M.; Emsley, L. J. Am. Chem. Soc. 2010, 132, 8252.

(16) Krushelnitsky, A.; Zinkevich, T.; Reichert, D.; Chevelkov, V.; Reif, B. J. Am. Chem. Soc. 2010, 132, 11850.

(17) Lewandowski, J. R.; Sass, H. J.; Grzesiek, S.; Blackledge, M.; Emsley, L. J. Am. Chem. Soc. 2011, 133, 16762.

(18) Tollinger, M.; Sivertsen, A. C.; Meier, B. H.; Ernst, M.; Schanda, P. J. Am. Chem. Soc. 2012, 134, 14800.

(19) Ma, P.; Haller, J. D.; Zajakala, J.; Macek, P.; Sivertsen, A. C.; Willbold, D.; Boisbouvier, J.; Schanda, P. Angew. Chem. Int. Ed. 2014, 53,4312 .

(20) Lamley, J. M.; Lougher, M. J.; Sass, H. J.; Rogowski, M.; Grzesiek, S.; Lewandowski, J. R. Phys. Chem. Chem. Phys. 2015, 17, 21997.

(21) Asami, S.; Porter, J. R.; Lange, O. F.; Reif, B. J. Am. Chem. Soc. 2015, 137, 1094.

(22) Knight, M. J.; Pell, A. J.; Bertini, I.; Felli, I. C.; Gonnelli, L.; Pierattelli, R.; Herrmann, T.; Emsley, L.; Pintacuda, G. Proc. Natl. Acad. Sci. USA 2012, 109, 11095.

(23) Lamley, J. M.; Iuga, D.; Oester, C.; Sass, H.-J.; Rogowski, M.; Oss, A.; Past, J.; Reinhold, A.; Grzesiek, S.; Samoson, A.; Lewandowski, J. R. J. Am. Chem. Soc. 2014, 136, 16800.

(24) Lamley, J. M.; Öster, C.; Stevens, R. A.; Lewandowski, J. R. Angew. Chem. Int. Ed. 2015, 54, 15374.

(25) Lewandowski, J. R.; Halse, M. E.; Blackledge, M.; Emsley, L. Science 2015, 348, 578.

(26) Ma, P. X.; Xue, Y.; Coquelle, N.; Haller, J. D.; Yuwen, T. R.; Ayala, I.; Mikhailovskii, O.; Willbold, D.; Colletier, J. P.; Skrynnikov, N. R.; Schanda, P. Nature Comm. 2015, 6.

(27) Good, D. B.; Wang, S. L.; Ward, M. E.; Struppe, J.; Brown, L. S.; Lewandowski, J. R.; Ladizhansky, V. J. Am. Chem. Soc. 2014, 136, 2833.

(28) Denisov, I. G.; Sligar, S. G. Nat. Struct. Mol. Biol. 2016, 23, 481.

(29) Huang, R.; Yamamoto, K.; Zhang, M.; Popovych, N.; Hung, I.; Im, S. C.; Gan, Z. H.; Waskell, L.; Ramamoorthy, A. Biophys. J. 2014, 106, 2126.

(30) Yamamoto, K.; Durr, U. H. N.; Xu, J. D.; Im, S. C.; Waskell, L.; Ramamoorthy, A. Sci. Rep. 2013, 3, 2538.

(31) Renault, M.; Saurel, O.; Czaplicki, J.; Demange, P.; Gervais, V.; Lohr, F.; Reat, V.; Piotto, M.; Milon, A. J. Mol. Biol. 2009, 385, 117.

(32) Westerlund, B.; Korhonen, T. K. Mol. Microbiol. 1993, 9, 687.

(33) Confer, A. W.; Ayalew, S. Vet. Microbiol. 2013, 163, 207.

(34) Lo, R. Y. C.; Sorensen, L. S. FEMS Microbiol. Lett. 2007, 274, 226.

(35) Andreas, L. B.; Le Marchand, T.; Jaudzems, K.; Pintacuda, G. J. Magn. Reson. 2015, 253, 36.

(36) Boeckmann, A.; Ernst, M.; Meier, B. H. J. Magn. Reson. 2015, 253,71 .

(37) Knight, M. J.; Webber, A. L.; Pell, A. J.; Guerry, P.; BarbetMassin, E.; Bertini, I.; Felli, I. C.; Gonnelli, L.; Pierattelli, R.; Emsley, L.; Lesage, A.; Herrmann, T.; Pintacuda, G. Angew. Chem. Int. Ed. Engl. 2011, 50, 11697.

(38) Nieuwkoop, A. J.; Franks, W. T.; Rehbein, K.; Diehl, A.; Akbey, U.; Engelke, F.; Emsley, L.; Pintacuda, G.; Oschkinat, H. J. Biomol. NMR 2015, 61, 161. 
(39) Barbet-Massin, E.; Pell, A. J.; Retel, J. S.; Andreas, L. B.; Jaudzems, K.; Franks, W. T.; Nieuwkoop, A. J.; Hiller, M.; Higman, V.; Guerry, P.; Bertarello, A.; Knight, M. J.; Felletti, M.; Le Marchand, T.; Kotelovica, S.; Akopjana, I.; Tars, K.; Stoppini, M.; Bellotti, V.; Bolognesi, M.; Ricagno, S.; Chou, J. J.; Griffin, R. G.; Oschkinat, H.; Lesage, A.; Emsley, L.; Herrmann, T.; Pintacuda, G. J. Am. Chem. Soc. 2014, 136, 12489.

(40) Wang, S.; Parthasarathy, S.; Xiao, Y.; Nishiyama, Y.; Long, F.; Matsuda, I.; Endo, Y.; Nemoto, T.; Yamauchi, K.; Asakura, T.; Takeda, M.; Terauchi, T.; Kainosho, M.; Ishii, Y. Chem. Commun. 2015, 51, 15055 .

(41) Eddy, M. T.; Su, Y.; Silvers, R.; Andreas, L.; Clark, L.; Wagner, G.; Pintacuda, G.; Emsley, L.; Griffin, R. G. J. Biomol. NMR 2015, 61, 299.

(42) Huber, M.; Hiller, S.; Schanda, P.; Ernst, M.; Boeckmann, A.; Verel, R.; Meier, B. H. Chem. Phys. Chem. 2011, 12, 915.

(43) Agarwal, V.; Penzel, S.; Szekely, K.; Cadalbert, R.; Testori, E.; Oss, A.; Past, J.; Samoson, A.; Ernst, M.; Boeckmann, A.; Meier, B. H. Angew. Chem. Int. Ed. 2014, 53, 12253.

(44) Barbet-Massin, E.; Huang, C.-T.; Daebel, V.; Hsu, S.-T. D.; Reif, B. Angew. Chem. Int. Ed. 2015, 54, 4367.

(45) Dannatt, H. R. W.; Felletti, M.; Jehle, S.; Wang, Y.; Emsley, L.; Dixon, N. E.; Lesage, A.; Pintacuda, G. Angew. Chem. Int. Ed. 2016, $55,6637$.

(46) Smith, A. A.; Testori, E.; Cadalbert, R.; Meier, B. H.; Ernst, M. J. Biomol. NMR 2016, 65, 1.

(47) Andreas, L. B.; Jaudzems, K.; Stanek, J.; Lalli, D.; Bertarello, A.; Le Marchand, T.; Paepe, D. C. D.; Kotelovica, S.; Akopjana, I.; Knott, B.; Wegner, S.; Engelke, F.; Lesage, A.; Emsley, L.; Tars, K.; Herrmann, T.; Pintacuda, G. Proc. Natl. Acad. Sci. USA 2016, 113, 9187.

(48) Mroue, K. H.; Nishiyama, Y.; Pandey, M. K.; Gong, B.; McNerny, E.; Kohn, D. H.; Morris, M. D.; Ramamoorthy, A. Sci. Rep. 2015, 5, 11991

(49) Nishiyama, Y. Solid State Nucl. Magn. Reson. 2016, 78, 24.

(50) Penzel, S.; Smith, A. A.; Agarwal, V.; Hunkeler, A.; Org, M. L.; Samoson, A.; Bockmann, A.; Ernst, M.; Meier, B. H. J. Biomol. NMR 2015, 63, 165 .
(51) Stanek, J.; Andreas, L. B.; Jaudzems, K.; Cala, D.; Lalli, D.; Bertarello, A.; Schubeis, T.; Akopjana, I.; Kotelovica, S.; Tars, K.; Pica, A.; Leone, S.; Picone, D.; Xu, Z. Q.; Dixon, N. E.; Martinez, D.; Berbon, M.; El Mammeri, N.; Noubhani, A.; Saupe, S.; Habenstein, B.; Loquet, A.; Pintacuda, G. Angew. Chem. Int. Ed. Engl. 2016, 55, 15504. (52) Schanda, P.; Meier, B. H.; Ernst, M. J. Magn. Reson. 2011, 210, 246.

(53) Park, S. H.; Yang, C.; Opella, S. J.; Mueller, L. J. J. Magn. Reson. 2013, 237, 164

(54) Iordanov, I.; Renault, M.; Reat, V.; Bosshart, P. D.; Engel, A.; Saurel, O.; Milon, A. Biochim. Biophys. Acta 2012, 1818, 2344.

(55) Rigaud, J. L.; Paternostre, M. T.; Bluzat, A. Biochemistry 1988, 27, 2677.

(56) Gullion, T.; Schaefer, J. J. Magn. Reson. 1989, 81, 196.

(57) Banigan, J. R.; Gayen, A.; Traaseth, N. J. Biochim. Biophys. Acta 2015, 1848, 334.

(58) Bremi, T.; Bruschweiler, R.; Ernst, R. R. J. Am. Chem. Soc. 1997, 119,4272

(59) Lewandowski, J. R.; Sein, J.; Blackledge, M.; Emsley, L. J. Am. Chem. Soc. 2010, 132, 1246.

(60) Columbus, L. Curr. Opin. Struct. Biol. 2015, 32, 131.

(61) Fox, D. A.; Columbus, L. Prot. Science 2013, 22, 1133.

(62) Bender, M. L.; Kezdy, J. Annu. Rev. Biochem. 1965, 34, 49.

(63) Fontana, A.; Fassina, G.; Vita, C.; Dalzoppo, D.; Zamai, M.; Zambonin, M. Biochemistry 1986, 25, 1847.

(64) Popot, J. L.; Trewhella, J.; Engelman, D. M. EMBO J. 1986, 5, 3039.

(65) Huang, K. S.; Bayley, H.; Liao, M. J.; London, E.; Khorana, H. G. J. Biol. Chem. 1981, 256, 3802.

(66) Rasko, D. A.; Rosovitz, M. J.; Myers, G. S.; Mongodin, E. F.; Fricke, W. F.; Gajer, P.; Crabtree, J.; Sebaihia, M.; Thomson, N. R.; Chaudhuri, R.; Henderson, I. R.; Sperandio, V.; Ravel, J. J. Bacteriol. 2008, 190, 6881 . 
SYNOPSIS TOC.

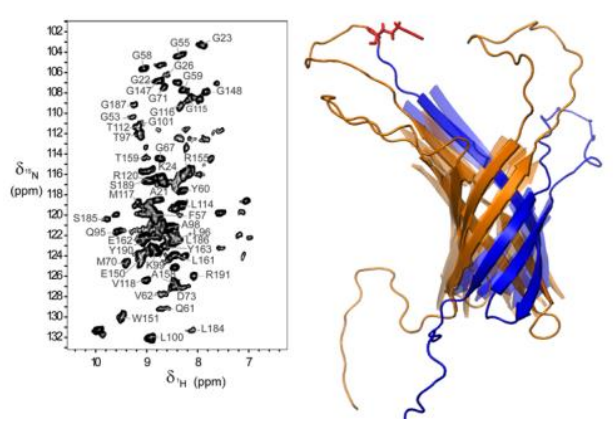

